University of Wollongong

Research Online

Faculty of Engineering and Information

Faculty of Engineering and Information

Sciences - Papers: Part A

Sciences

$1-1-2014$

Neutron-gamma pulse shape discrimination using organic scintillators with silicon photomultiplier readout

Rhys M. Preston

University of Wollongong, rmp987@uowmail.edu.au

John Eberhardt

CSIRO

James Tickner

CSIRO

Follow this and additional works at: https://ro.uow.edu.au/eispapers

Part of the Engineering Commons, and the Science and Technology Studies Commons

Research Online is the open access institutional repository for the University of Wollongong. For further information contact the UOW Library: research-pubs@uow.edu.au 


\title{
Neutron-gamma pulse shape discrimination using organic scintillators with silicon photomultiplier readout
}

\author{
Abstract \\ Neutron/Gamma pulse shape discrimination (PSD) was measured using stilbene and EJ-299-34 plastic \\ scintillators with readout by silicon photomultipliers (SiPMs). The SiPM pulses were digitized and \\ processed for energy and pulse shape information using a digital technique performing numerical \\ weighted integrations on each pulse. A genetic algorithm (GA) was developed to optimize the weighting \\ vectors used for the pulse shape discrimination. Efficient PSD was obtained down to an electron- \\ equivalent energy of $127 \mathrm{keV}$ with stilbene and $391 \mathrm{keV}$ with the EJ-299-34 PSD plastic. Separation at \\ lower energies was possible at reduced detection efficiency, down to $78 \mathrm{keV}$ and $186 \mathrm{keV}$ at $50 \%$ \\ efficiency. The detectors were used to measure separated gamma and neutron spectra from an \\ Americium-Beryllium neutron source and from a Na-22 gamma source. The GA-optimized weighted \\ integration was compared with digital charge comparison (DCC). The GA exhibited slightly improved \\ performance with a $400 \mathrm{MSps}$ digitization rate and showed a significant advantage at sample rates below \\ 100 MSps.

\section{Keywords} \\ discrimination, organic, scintillators, silicon, photomultiplier, readout, neutron, pulse, gamma, shape \\ Disciplines \\ Engineering | Science and Technology Studies

\section{Publication Details} \\ Preston, R. M., Eberhardt, J. E. \& Tickner, J. R. (2014). Neutron-gamma pulse shape discrimination using \\ organic scintillators with silicon photomultiplier readout. IEEE Transactions on Nuclear Science, 61 (4), \\ 2410-2418.
}




\title{
Neutron-Gamma Pulse Shape Discrimination using Organic Scintillators with Silicon Photomultiplier Readout
}

\author{
Rhys M. Preston, Student Member, IEEE, John E. Eberhardt, and James R. Tickner
}

\begin{abstract}
Neutron/Gamma pulse shape discrimination (PSD) was measured using stilbene and EJ-299-34 plastic scintillators with readout by silicon photomultipliers (SiPMs). The SiPM pulses were digitized and processed for energy and pulse shape information using a digital technique performing numerical weighted integrations on each pulse. A genetic algorithm (GA) was developed to optimize the weighting vectors used for the pulse shape discrimination. Efficient PSD was obtained down to an electron-equivalent energy of $127 \mathrm{keV}$ with stilbene and $391 \mathrm{keV}$ with the EJ-299-34 PSD plastic. Separation at lower energies was possible at reduced detection efficiency, down to $78 \mathrm{keV}$ and $186 \mathrm{keV}$ at $50 \%$ efficiency. The detectors were used to measure separated gamma and neutron spectra from an Americium-Beryllium neutron source and from a Na-22 gamma source. The GA-optimized weighted integration was compared with Digital Charge Comparison (DCC). The GA exhibited slightly improved performance with a $400 \mathrm{MSps}$ digitization rate and showed a significant advantage at sample rates below 100 MSps.
\end{abstract}

Index Terms- Genetic algorithms, neutron spectroscopy, pulse shape discrimination, scintillation detectors, silicon photomultiplier.

\section{INTRODUCTION}

$\mathrm{T}$ HE silicon photomultiplier (SiPM) is a solid-state photo detector that has established itself as a viable alternative to traditional vacuum photomultiplier tubes (PMTs) in many applications including high energy physics [1], homeland security [2] and medical imaging [3]. The SiPM is based on a matrix of avalanche photodiode micro-pixels operating in a self-quenched Geiger-mode [4], [5]. These micro-pixels are connected in parallel to form a quasi-analogue photo-detector. The SiPM provides high gain and photo-detection efficiency (PDE) similar to that of a typical PMT, with the ruggedness, low cost and small size associated with solid-state devices.

Manuscript received August 14, 2013; revised January 28, 2014; accepted June 27, 2014.

R. Preston is with the CSIRO Mineral Resources National Research Flagship, Lucas Heights, Australia; and the Centre for Medical Radiation Physics, University of Wollongong, Wollongong, Australia (e-mail: rhys.preston@csiro.au).

J. Eberhardt and J. Tickner are with the CSIRO Mineral Resources National Research Flagship, Lucas Heights, Australia (e-mail: john.eberhardt@csiro.au; james.tickner@csiro.au).
We are investigating the application of silicon photomultipliers to fast neutron detection [6], [7]. Organic scintillator detectors are widely used for the online detection of fast neutrons, using scintillations produced by proton recoil [8]. Organic scintillators are also sensitive to gamma radiation, so gamma-ray induced scintillations may present a background that limits the effective measurement of fast neutron flux.

In some types of organic crystal, liquid and plastic scintillators, the temporal profile of the scintillation photon emission depends on the linear energy transfer (LET) of the ionizing particle [8]. The scintillation time profile (pulseshape) may be used to determine the type of incoming ionizing particle. This technique is known as pulse shape discrimination (PSD). One common use of PSD is for the separated measurement of mixed neutron / gamma fields, by discriminating scintillations from high-energy electrons produced in collisions with gamma-rays from those due to neutron-scattered recoil-nuclei (heavy charged particles).

Traditionally photomultipliers (PMTs) are used for PSD, being well suited for the measurement of the scintillation time profiles. However, their inherent large size, cost, sensitivity to magnetic fields and high voltage requirements limit the wider application of the PSD technique. Some of those difficulties have been compensated by using flat-panel PMTs [9]. Using an SiPM for the optical readout of the scintillations makes a detector better fitted for use in hand-held instruments and in applications using large, granular detector arrays. However, the SiPM exhibits some characteristics that present a challenge for precise electronic readout. These include a longer singlephoton response duration and noise contributions from dark pulses, after-pulsing and crosstalk.

The single-photon response (SPR) describes the pulse shape produced by a photo-detector following the detection of a single photon. In a PMT, the SPR can be relatively narrow; the shape arises primarily from the spread in transit times of electrons travelling through the tube, and the subsequent discharge of the anode through the external load [10]. For a timing PMT and suitable signal chain, the SPR may exhibit a FWHM of a few ns. Conversely, the charge generated by the Geiger avalanche in an SiPM micro-pixel must flow through the passive network formed by the SiPM and analogue frontend [11], [12]. Depending on the design of the SiPM and the 
readout circuit, a typical single photon response exhibits a 1-20 ns rising edge and a slower 10-100 ns falling edge with one or more characteristic time constants.

SiPM dark pulses are thermally triggered pulses, identical in shape to those generated by the detection of a photon. These dark pulses are produced at rates between $10^{5}$ and $10^{6}$ counts per second (cps) per $\mathrm{mm}^{2}$ of active area, compared to $<1 \mathrm{cps}$ per $\mathrm{mm}^{2}$ for a modern PMT. Dark pulses are a persistent source of baseline disturbance, piling-up with scintillation events and other dark pulses. Example dark pulses, measured from a $1 \mathrm{~mm}^{2}$ SensL SPMMicro1100 SiPM, are shown in Fig. 1. The SiPM anode was connected to the $50 \Omega$ input of a FEMTO HVA-500M-20-B amplifier with $\times 10$ voltage gain. The dark pulses exhibit the SPR characteristics of the SiPM.

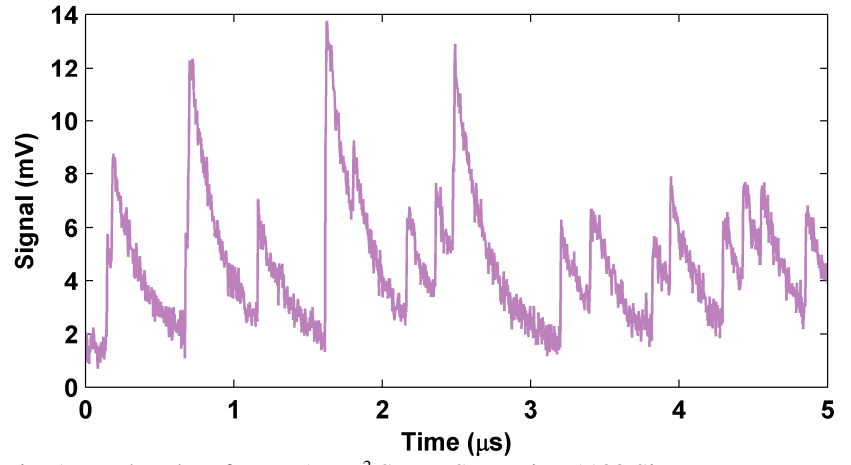

Fig. 1. Dark pulses from a $1 \mathrm{~mm}^{2}$ SensL SPMMicro1100 SiPM.

Crosstalk and after-pulsing are a function of the SiPM design and multiply the response of signals from some triggered pixels (both thermally and optically triggered), contributing another source of excess noise [13]. Crosstalk is due to the emission of optical photons during avalanche, with some photons triggering neighboring micro-pixels. Afterpulsing is due to the trapping and eventual release of avalanche carriers, sometimes re-triggering the recharging micro-pixel.

Fig. 2 shows pulse height spectra measured from a fastneutron detector comprising of the abovementioned SPMMicro1100 SiPM coupled to a $1 \mathrm{~cm} \times 1 \mathrm{~cm} \times 5 \mathrm{~cm}$ EJ-204 plastic scintillator. The amplifier output was digitized, with the pulse heights processed in software. The fast-neutron spectrum was collected with the detector exposed to $14 \mathrm{MeV}$ neutrons from a deuterium-tritium (DT) fusion source, while the dark noise spectrum was measured in the absence of a radiation source. The first peak in both spectra corresponds to pulse height due to a single micro-pixel firing, while the subsequent peaks are due to multiple micro-pixels firing nearsynchronously. The DT spectrum includes scintillations produced by neutron-recoiled protons. The limited scintillation signal is attributed to the small SiPM area relative to the scintillator dimensions. Dark pulses dominate low-energy region of both spectra. Due to cross-talk a significant proportion of dark pulses ( $20 \%)$ have pulse heights above that of a single triggered micro-pixel.

Despite the noise associated with SiPMs, they have successfully been applied to performing $\alpha / \beta$ PSD with a liquid scintillator [14]. They have also been used to discriminate the triton and alpha products (due to neutron capture) from electrons (due to gammas) in CYLC inorganic crystals [15], [16]. Current PSD methods have been designed for the processing of PMT signals that in large part lack the features associated with SiPMs. We are developing digital pulse processing (DPP) techniques and hardware for the measurement of PSD using SiPM readout [7]. With this processing tailored for use with SiPMs, it is anticipated that SiPM-based PSD detectors may approach the performance of those based on PMT readout.

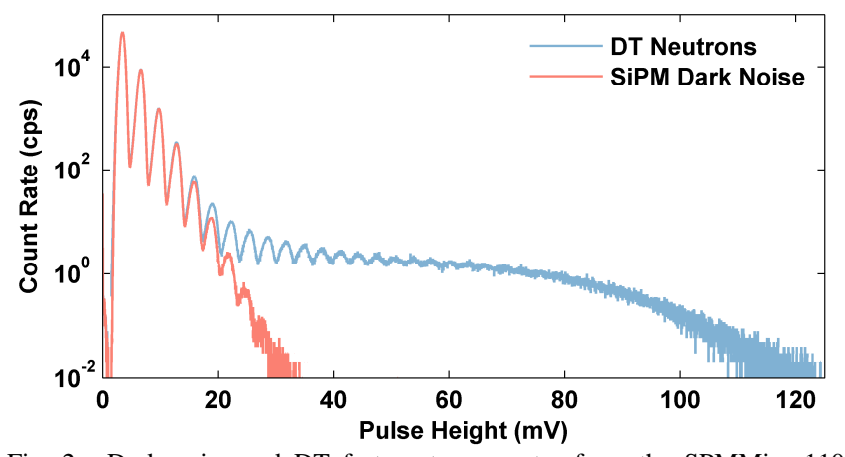

Fig. 2. Dark noise and DT fast-neutron spectra from the SPMMicro1100 SiPM coupled to an EJ-204 plastic scintillator.

\section{Digital Methods for Pulse Shape Discrimination}

The organic scintillators used for PSD include organic crystals, liquid scintillators [17] and recently developed plastic scintillators [18]. In these scintillators the proportion of fast ( $\sim \mathrm{ns}$ and $\sim 10 \mathrm{~ns})$ components to the slower ( 100 ns) components of the scintillation decay is reduced for high LET particles. Most PSD processing techniques derive some parameter $S$, indicating the shape of each pulse. Gamma and neutron events produce pulses with differing $S$ values and so the scintillations may be classified by placing cuts on $S$.

The PSD figure of merit (FOM) is used to quantify the performance of a PSD setup at a given energy. This FOM is commonly assessed from the full-width-half-maximum (FWHM) and mean $(\mu)$ of the neutron (n) and gamma $(\gamma)$ peaks in the $S$ histogram for a chosen energy range [17]

$$
F O M=\frac{\left|\mu_{\gamma}-\mu_{n}\right|}{F W H M_{\gamma}+F W H M_{n}} .
$$

The FOM indicates the relative overlap between the $S$ distributions measured from each particle type. Higher figures of merit allow a greater proportion of pulses to be correctly classified. With many PSD techniques, the $S$ distributions are approximately Gaussian. In this case the expected rate of pulses being misclassified may be calculated using the FOM, relative distribution widths and cut thresholds [19]. A FOM above 1.27 has been used to indicate efficient PSD [18]. This is the FOM where the means of two Gaussian peaks are separated by $3 \times$ the sum of their standard deviations $\sigma$ $(\mathrm{FWHM} \approx 2.35 \sigma)$.

PSD pulse processing was initially performed using 
analogue signal chains, with techniques based on linear filtering [20], zero-crossing [17] and charge comparison [21]. PSD processing may also be implemented in the digital domain, where the detector or preamplifier signals are directly sampled using an analogue-to-digital convertor (ADC). The digital representation of the signals is then processed using a microprocessor or field programmable gate array (FPGA), often alongside energy and timing information. Digital pulse processing (DPP) solutions typically offer superior flexibility, as the processing algorithms may be tuned or altered without changes to the hardware.

DPP algorithms for PSD measurements have been developed with varying degrees of sophistication and computing complexity. Some techniques include correlation of pulses with standard pulse shapes [22], parameterized fitting [23], comparing sample heights at the pulse peak and on the trailing edge [24], utilizing artificial neural networks [25], and the analysis of pulses in the frequency domain using wavelet transforms [26], [27] and Fourier transforms [28].

\section{A. Digital Pulse Processing using Weighted Integration}

The digital pulse processing implemented in this work relies on weighted numerical integrations of each pulse for determining both energy $E$ and pulse shape $S$ :

$$
S=\sum_{i=1}^{n} w_{i} \cdot p_{i}
$$

where the $n$ samples of each digitized pulse $\boldsymbol{p}$ are multiplied by corresponding coefficients in a weighting vector $\boldsymbol{w}$ aligned with the pulse and accumulated to give the measured result. By balancing $w$, such that the sum of the coefficients is zero, the integration is made insensitive to DC shifts in the signal baseline. In the following measurements, $\boldsymbol{p}$ represents the voltage over time produced by the SiPM with its anode connected to a $50 \Omega$ load resistor. However, the technique may just as easily be applied to the output of a preamplifier or shaping electronics [29].

For PSD, $\boldsymbol{w}$ needs to be set so $S$ best characterizes the scintillation time profile. Because $S$ is also proportional to the amplitude of the pulse, it is normalized using the measured energy $E$. The weighted integral presents a generalization of a number of already developed PSD algorithms. Digital Charge Comparison (DCC) is a commonly used technique that may be implemented as a weighted integration using rectangular coefficients with an integration window that is shorter or delayed with respect to the energy integration [30].

If the photo-detector SPR is short compared to the scintillation decay times the optimum $\boldsymbol{w}$ for PSD may be calculated analytically using average pulse shapes arising from neutrons and gammas [20]. This calculation has been adjusted to take account of background noise sources [31], and for the case where the pulse is charge-integrated before being sampled [29].

When using an SiPM rather than a PMT, the signal from the device less faithfully represents the time profile of the scintillation. Rather, the scintillation profile is convolved with the relatively slow SiPM SPR, shaping the output signal. For example, the SPR of the MicroFB-60035 SiPM used in this work had a $280 \mathrm{~ns}$ FWHM. In addition, there are the contributions due to dark pulses, crosstalk and after-pulsing. These factors complicate the analytical calculation of optimum pulse weights. The approach taken in this work was to use numerical optimization methods to estimate the optimum weighting coefficients for each SiPM-scintillator pairing. A genetic algorithm was developed for this purpose.

\section{B. Genetic Algorithm for the Optimization of Weighting Vectors}

The genetic algorithm (GA) is a search heuristic inspired by evolutionary 'survival of the fittest' models in nature and is based on combining and mutating generations of provisional solutions [32]-[34]. It is well suited to problems such as the search for an optimal set of weights; where the search space is large, not necessarily differentiable and may contain multiple local minima.

The GA works with a population of potential solutions typically referred to, by analogy with the biological world, as chromosomes. Each chromosome contains the set of parameters (genes) to be optimized, in this case the elements of the weighting vector $\boldsymbol{w}$. The first generation of solutions is randomly generated. The chromosomes are judged against performance criteria and awarded a fitness rating. Pairs of chromosomes (parents) are then selected to combine and produce daughter chromosomes in a process called crossover. The selection of parents for combination is done stochastically, with chromosomes of higher fitness given a higher probability of being chosen. The next generation is populated by these newly generated daughter chromosomes, which undergo some random mutation before the process repeats.

In the canonical GA [32], the mutation and cross-over operations directly manipulate the binary bit strings representing the chromosome parameters. Mutations flip bits while crossover produces offspring by truncating and splicing the parents at a random position in the bit string. The GA developed for this work instead manipulates the integer encoding of the genomes [35], with the integer range of the weights being user-adjustable. The real-coded approach was taken so that the balancing constraint on the weighting vectors could be readily enforced.

The genetic algorithm was implemented as a MATLAB (Mathworks, MA, USA) script, with a population size of 100. To optimize the PSD $\boldsymbol{w}$ for a particular detector, a training set of gamma and neutron pulses (previously identified with a traditional method, such as DCC) were fed to the GA. Each prospective $\boldsymbol{w}$ in the population was used to integrate the training pulses, producing a set of neutron and gamma $S$ values. The mean and standard deviation of the $S / E$ values were used to estimate the FOM measured with the training pulses. The assessed FOM was used to indicate the fitness of each $\boldsymbol{w}$ for the selection and cross-over process.

Selection was accomplished using a roulette wheel method, with the probability of selection inversely proportional to the 
$\boldsymbol{w}$ vector's rank in fitness [36]. Flat crossover was used for recombination, with each weight of the daughter randomly chosen from the interval defined by the corresponding weights of the parents [37]. Mutation was implemented by adding randomly chosen values to random weights on each vector. Each weight had a $1 \%$ chance of undergoing mutation, with the mutating value sampled from a discrete Gaussian distribution with zero mean and standard deviation equal to $5 \%$ the configured integer range. Modifications to a weight exceeding the allowed range saturated. Elitism allowed the best performing individual of each population to continue unaltered to the next generation [34], preventing the loss of the best performing $\boldsymbol{w}$ up to that point. Four randomly generated chromosomes were introduced to each generation in an effort to maintain genetic diversity. After each new generation was produced, the balancing constraint was imposed by incrementing (or decrementing as necessary) random weights until each chromosome was balanced. The GA cycle subsequently started over with the new generation.

\section{DeteCtor MAterials, CONSTRUCTION AND REAdout}

A primary motivation for this work was to investigate a compact neutron/gamma discriminating detector suitable for hand-held use. For this purpose, two types of PSD scintillator were obtained: $1 \mathrm{~cm} \times \varnothing 1 \mathrm{~cm}$ cylindrical stilbene single crystals from ScintiTech (MA, USA) and EJ-299-34 PSD plastic scintillators from Eljen Technology (TX, USA). The EJ-299-34 was a developmental-prototype based on the PSD plastic invented at Lawrence Livermore National Laboratory [18] and being commercialized by Eljen Technology [38]. The plastic was diamond milled into $1 \mathrm{~cm} \times 1 \mathrm{~cm} \times 5 \mathrm{~cm}$ bars.

Both the stilbene and the EJ-299-34 produce scintillation photons with wavelengths peaked around $\sim 400 \mathrm{~nm}$. SiPMs with a p-on-n structure have their peak PDE in the blue region of the spectrum and were best matched for the scintillations. Two commercially available p-on-n SiPMs were selected, a Hamamatsu S10985-050C and a SensL MicroFB-60035 (abbreviated as FB60035). Both SiPM types had a total active area of $\sim 36 \mathrm{~mm}^{2}$.

The stilbene crystals were coupled to the photo-detectors using GE RTV615 silicone. As the RTV615 failed to cure while in contact with the EJ-299-34, the EJ-299-34 detectors were coupled using an EJ-560 silicone pad instead. The scintillators were wrapped with $3 \mathrm{M}$ Vikuiti Enhanced Specular Reflector. Teflon tape was used to cover small gaps in the reflector. Example detectors using the FB60035 SiPMs are shown in Fig 3.

Printed circuit boards (PCBs) were made to reliably interface with the 5 pin ceramic package of the S10985-050C and the 4 pad surface-mount package of the FB60035. The PCBs included a decoupling network to provide a stiff bias voltage. This consisted of a $2 \mathrm{k} \Omega$ series resistor followed by a pair of capacitors ( $1 \mu \mathrm{F}$ and $100 \mathrm{nF})$ to ground. The scintillator side of the PCB was painted with titanium dioxide white paint to form part of the reflector. The SiPMs were operated in a grounded tin-plated steel enclosure, with the anode signals and bias connected to BNC bulkhead sockets.
The S10985-050C SiPMs were operated at $71.34 \mathrm{~V}$ and $71.65 \mathrm{~V}$, as specified by Hamamatsu in their test sheets. The FB60035 SiPMs were biased at $27 \mathrm{~V}$, corresponding to $2.5 \mathrm{~V}$ above breakdown. All measurements were made at ambient temperature, which varied between $20^{\circ} \mathrm{C}$ and $22^{\circ} \mathrm{C}$.

The signals were sampled at $400 \mathrm{MSps}$ with $14 \mathrm{bit}$ resolution using a Signatec PX14400D2 digitizer with a DC$180 \mathrm{MHz}$ analogue front-end. The SiPM anode signals were connected to the $50 \Omega$ input of the digitizer using an RG-58 coaxial cable. The digitizer was set to collect traces of 2048 sample length, triggered by the rising edge of each pulse, and recorded them to the hard drive for later processing in software.

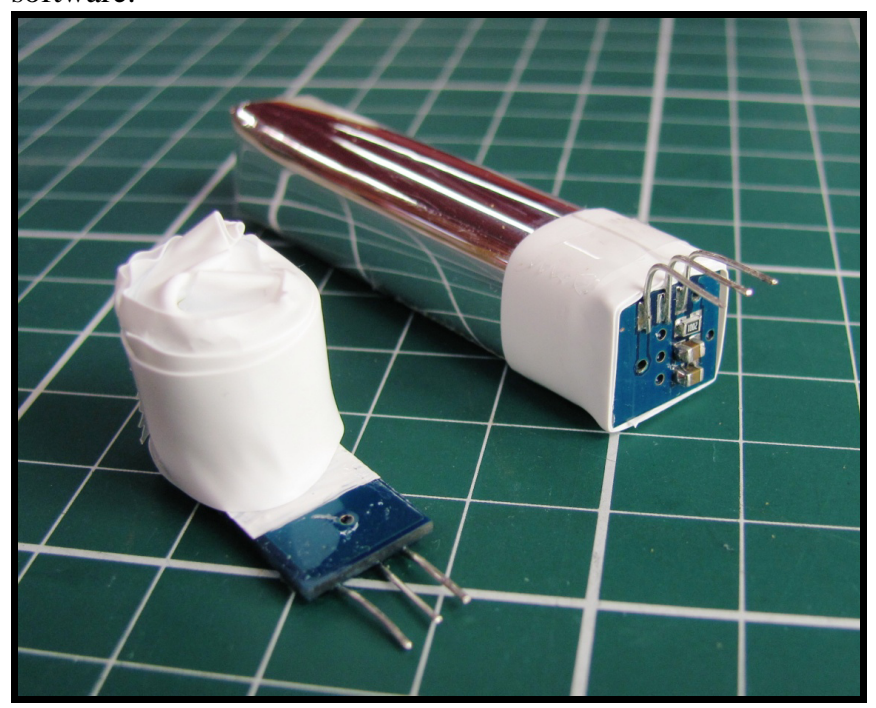

Fig 3. Stilbene (left) and EJ-299-34 (right) scintillator detectors with FB60035 SiPM readout. For scale, the small grid squares measure $1 \mathrm{~cm} \times 1 \mathrm{~cm}$.

To compare the performance of the SiPM-based detectors with those using PMT readout, the scintillators were also coupled to a Photonis XP2262 PMT. The PMT anode was connected to a custom preamplifier providing $1 \mu \mathrm{sC}$ shaping. Shaping provides a method to overcome the dynamic range limits of ADCs used for digital PSD techniques [29], [39]. The preamplifier output signals were digitized and processed alongside the SiPM data.

\section{MEASUREMENT}

The detectors were used to measure fast-neutrons from an Americium-241 Beryllium (AmBe) radio-isotope neutron source. The AmBe source produces a broad spectrum of fast neutrons with energies up to $10 \mathrm{MeV}$ [40] and a gamma spectrum with peaks up to $4.4 \mathrm{MeV}$ [41]. An additional gamma background was expected, produced in neutron interactions with the surrounding environment. As the collection of gamma and neutron pulses in a broad range energy range was desired for FOM assessment, no attempt was made to shield the detectors from gammas. The distance between each detector and source was adjusted to keep the count-rate below $1 \mathrm{kcps}$, limiting pulse pile-up.

The detectors were also used to measure a Na-22 source. 
Organic scintillators exhibit different light yield responses to electrons and protons [42]. The $341 \mathrm{keV}$ and $1057 \mathrm{keV}$ Compton edges in the Na-22 gamma spectrum, arising from the $511 \mathrm{keV}$ and $1275 \mathrm{keV}$ gamma-rays emitted by the source, were used to calibrate the energy scale in units of electronequivalent $\mathrm{eV}\left(\mathrm{eV}_{\mathrm{ee}}\right)$. The position of each Compton edge was estimated at half-height.

The digitized pulses were processed using a MATLAB script. Each pulse was aligned according to the sample that first exceeded $50 \%$ of the relative height above baseline. The energy and PSD weighted integrations were performed by taking the dot product of the aligned pulse with each weighting vector. The energy was integrated for $2 \mu \mathrm{s}$, with a $1 \mu \mathrm{s}$ baseline window preceding the pulse to balance the weighting vector.

\section{A. Genetic Algorithm and DCC Optimization}

The $S$ values were initially calculated using a non-optimized set of DCC weights, integrating the pulse in a time window shorter than the energy measurement. The arbitrary DCC scheme provided sufficient PSD to reliably separate neutron and gamma events at $500 \mathrm{keV}_{\text {ee }}$ and above. AmBe scintillations between $500 \mathrm{keV}_{\mathrm{ee}}$ and $600 \mathrm{keV}_{\mathrm{ee}}$, identified as either gamma or neutron pulses, were used to form a training set for the GA. Each training set had at least five thousand gamma and neutron pulses.

The GA was used to generate optimum weighting vectors for each detector setup. Fig. 4 shows the best figures of merit measured over 10 thousand generations for three independent runs of the GA using the stilbene FB60035 training sets. After a thousand generations, the various independent runs are near convergence. Using ten thousand pulses in each training bank, the GA took 21 minutes to complete 1000 generations for 2048 sample pulses and 4 minutes for 256 sample pulses on a standard desktop computer (Intel i5-2400 processor). The generated $\boldsymbol{w}$ vectors showed some statistical fluctuations, so the results from multiple GA runs were averaged to reduce these. There was no measurable change in PSD performance between the original and averaged vectors.

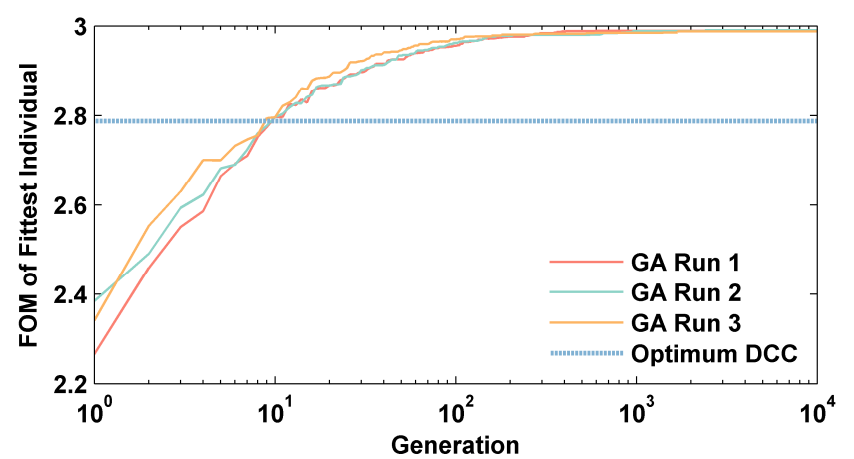

Fig. 4. Improvement of FOM measured from the fittest individual in each generation of the GA for the stilbene FB60035 detector at $\sim 550 \mathrm{keV}_{\mathrm{ee}}$. Also shown is the best FOM achieved using the optimized DCC method.

An example $\boldsymbol{w}$ is shown in Fig. 5. The GA-optimized $\boldsymbol{w}$ vectors for each detector had similar characteristics, the dominant trait being a region of positive weights covering the first $\sim 100 \mathrm{~ns}$ of the pulse. The underlying neutron and gamma scintillation profiles, normalised using the $2 \mu \mathrm{s} E$ integration, exhibit the greatest disparity in this time period. The analytically derived optimum $\boldsymbol{w}$ vectors for charge-integrated pulses share a similar feature [29]. The preceding weights, aligned with the baseline, act to balance the $w$ vector.

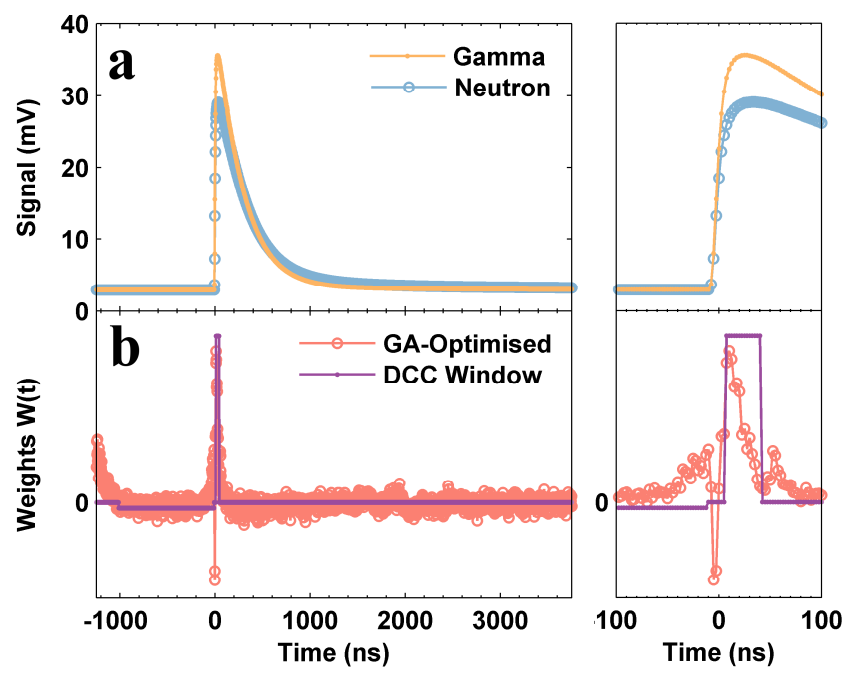

Fig. 5. a) Average $\sim 550 \mathrm{keV}$ neutrons and gamma pulse shapes measured with the EJ-299-34 FB60035 detector. b) Corresponding GA-optimized weights and optimum DCC integration window. The right plots show a zoomed view of the pulse rising edge.

The training banks were also used to determine the optimum DCC window for each detector. The baseline window was locked to the same $1 \mu$ s window as used for the energy measurement. A 2-parameter scan, changing the DCC window start and stop times, was performed. The optimized DCC window approximated the GA-generated weights by placing a short window towards the beginning of the pulse. The optimum DCC window durations for the various detectors were between $15 \mathrm{~ns}$ and $50 \mathrm{~ns}$.

The optimal DCC and GA-optimized weighting vectors were then used to re-process the $\mathrm{AmBe}$ and $\mathrm{Na}-22$ datasets for the assessment of PSD performance and neutron/gamma separation. The subset of pulses used for GA training was excluded from each dataset during the FOM evaluation.

\section{B. PSD Performance}

A MATLAB script was written to evaluate the PSD figures of merit as measured by each detector in the energy range between $25 \mathrm{keV}_{\text {ee }}$ and $2.5 \mathrm{MeV}_{\text {ee }}$. The script took $25 \mathrm{keV}_{\text {ee }}$ wide energy slices of the data and generated a histogram of the $S / E$ values. The $S / E$ histograms were fitted with a sum of two Gaussian curves using a weighted least-squares, constrained, nonlinear minimization. The model was found to provide a good description of the data. The FOM was then calculated using the FWHM and mean $\mu$ of the fitted curves. The evaluation was performed using the AmBe data. Consequently, the figures of merit shown in Fig. 6 and Fig. 7 reflect the performance of the system with the digitizer input range suitable for capturing scintillations of energies up to $\sim 5 \mathrm{MeV}_{\text {ee. }}$. 


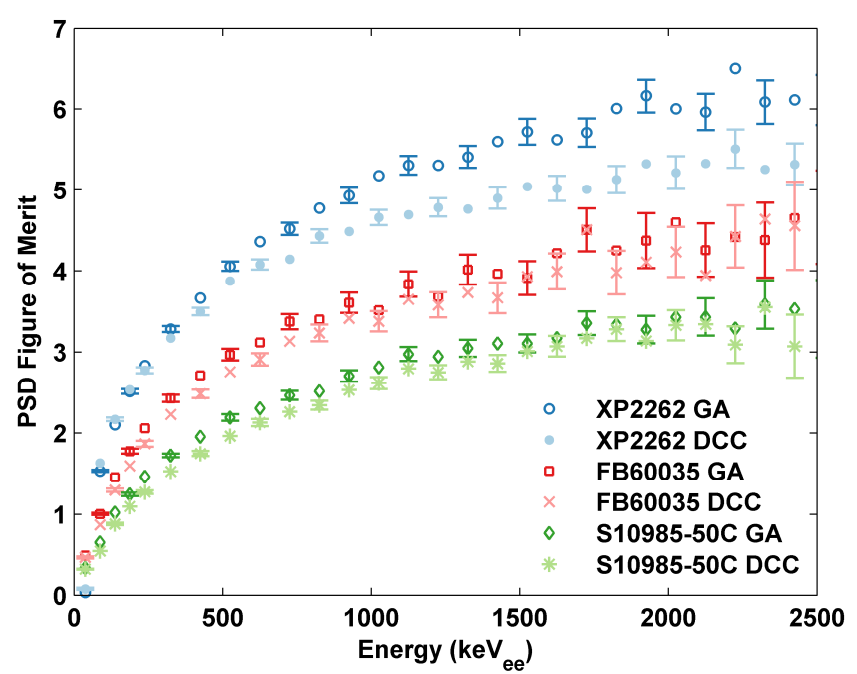

Fig. 6. PSD Figures of Merit measured with the stilbene single crystal detectors using DCC and GA-optimized weighted integration. For clarity, only every second data point has its $\pm 2 \sigma$ error bars displayed.

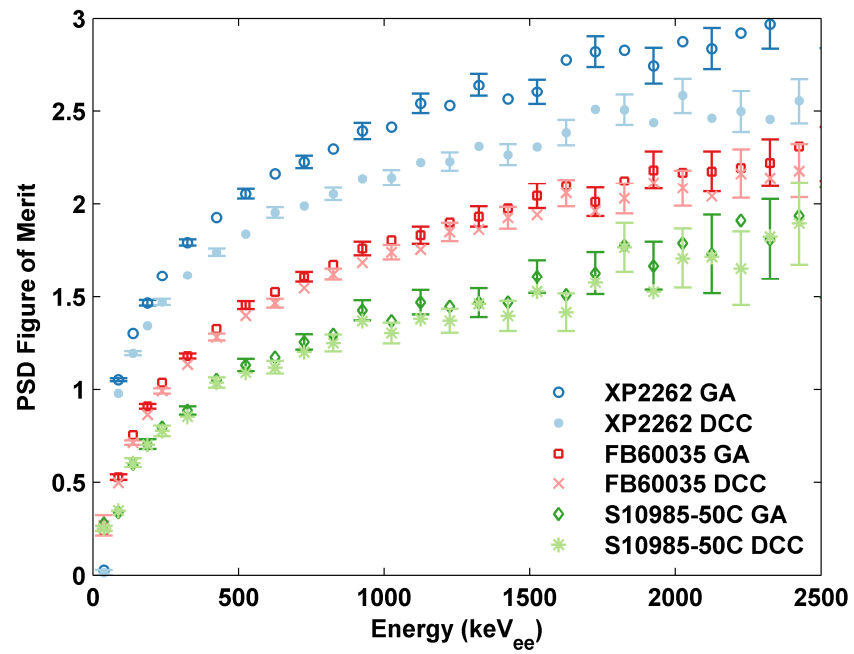

Fig. 7. PSD Figures of Merit measured with the EJ-299-34 detectors using DCC and GA-optimized weighted integration. For clarity, only every second data point has its $\pm 2 \sigma$ error bars displayed.

With each detector the GA-optimized weights provided figures of merit slightly higher than those with DCC processing. The small difference between the figures of merit suggests that DCC makes a reasonable approximation for the optimal weights with these detectors and digitization. This is consistent with previous measurements and simulations, where DCC provided performance near that of the analyticallyderived optimum weights [30], [39].

The XP2262 PMT provided the best figures of merit for both scintillators, followed by the FB60035 and finally the S10985-050C. The reason behind the reduced performance of the SiPM-detectors may require a detailed study of the excess noise characteristics and PDE of each SiPM type. With the FB60035-based detectors, efficient PSD was possible down to $127 \mathrm{keV}_{\text {ee }}$ with stilbene and $391 \mathrm{keV}_{\text {ee }}$ using the EJ-299-34. This is compared with $69 \mathrm{keV}_{\mathrm{ee}}$ and $130 \mathrm{keV}_{\mathrm{ee}}$ using the XP2262 PMT. The rest of this paper focuses on subsequent measurements made with the FB60035 detectors.

\section{Neutron-Gamma Discrimination}

In order to produce separate neutron and gamma spectra, $S / E$ thresholds were set to classify each scintillation event as a neutron or gamma, or reject pulses where the measured shape was ambiguous. Rejecting pulses reduces the effective detection efficiency of the detector. The acceptance ratio describes the proportion of processed pulses classified as a neutron or gamma at a given energy. Meanwhile, the misclassification rate describes the proportion of pulses being incorrectly classified, i.e. a neutron being identified as a gamma or vice-versa. Setting the $S / E$ thresholds involves making a balance between the acceptance ratio and the misclassification rate.

The parameters of the Gaussian fits, used previously for FOM assessment, were used to set gamma and neutron $S / E$ cuts as a function of energy. The cuts were set according to the $\mu \pm 3 \sigma$ boundaries of the respective neutron and gamma $S / E$ distributions. The conditions for a scintillation to be classified as a neutron were:

$$
\begin{aligned}
& \left(\mu_{n}(E)-3 \sigma_{n}(E)\right)<S / E<\left(\mu_{n}(E)+3 \sigma_{n}(E)\right) \text { and } \\
& S / E<\left(\mu_{\gamma}(E)-3 \sigma_{\gamma}(E)\right) .
\end{aligned}
$$

Likewise, for the pulse to be classified as a gamma:

$$
\begin{aligned}
& \left(\mu_{\gamma}(E)-3 \sigma_{\gamma}(E)\right)<S / E<\left(\mu_{\gamma}(E)+3 \sigma_{\gamma}(E)\right) \text { and } \\
& S / E>\left(\mu_{n}(E)+3 \sigma_{n}(E)\right) .
\end{aligned}
$$

The calculated $S / E$ boundaries for the FB60035 stilbene detector are shown in Fig. 8.

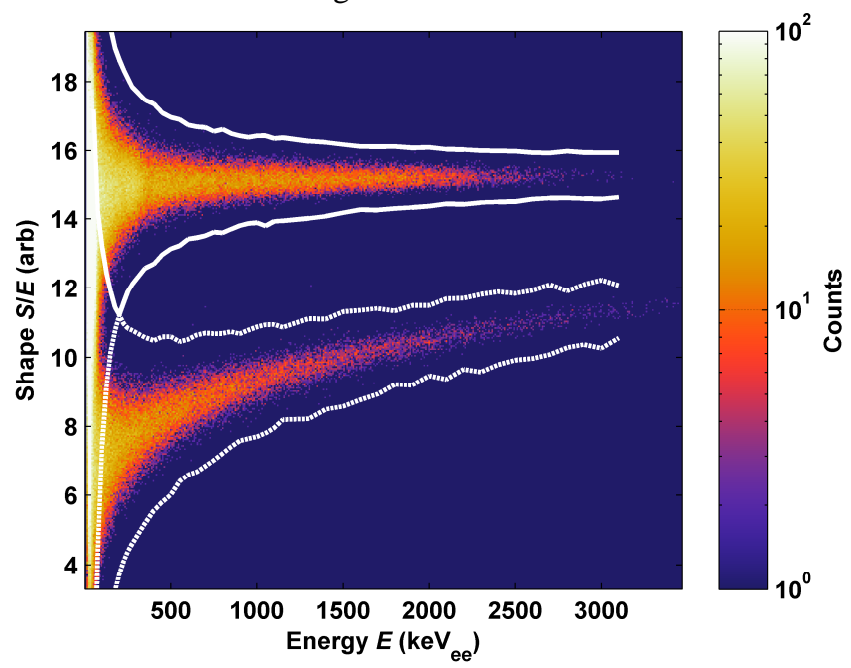

Fig. 8. Pulse shape and energy measured from the stilbene FB60035 detector exposed to an $A m B e$ source. The dashed lines show the set $S / E$ neutron cuts and the solid lines show the gamma cuts.

The adjacent $\mu_{\mathrm{n}}+3 \sigma_{\mathrm{n}}$ neutron and $\mu_{\mathrm{g}}-3 \sigma_{\mathrm{g}}$ gamma boundaries intersect when the FOM equals 1.27, the indicative threshold for efficient PSD. At higher energies, where FOM $>1.27$, the distributions are adequately separated so that (3) and (5) are solely responsible for classifying pulses and the 
acceptance ratio should be $\sim 99.7 \%$. At lower energies, where FOM $<1.27$, (4) and (6) act to limit the rate of misclassified pulses to $\sim 0.1 \%$. In this case, a greater proportion of pulses are rejected due to their ambiguous shape and the acceptance ratio is expected to fall.

Separated Na-22 and AmBe spectra measured with the EJ-299-34 FB60035 detector are shown in Fig. 9 and Fig. 10. The Na-22 gamma spectrum shows the $341 \mathrm{keV}$ and $1057 \mathrm{keV}$ Compton edges as well as some natural background at higher energies. The Na-22 neutron spectrum is made up of gamma events incorrectly classified as neutrons. The misclassification rate in each energy bin was below $0.1 \%$, as intended. The rejected spectra contain the pulses whose $S / E$ value fell outside the specified cuts. These rejected counts become significant at low energies due to the falling FOM.

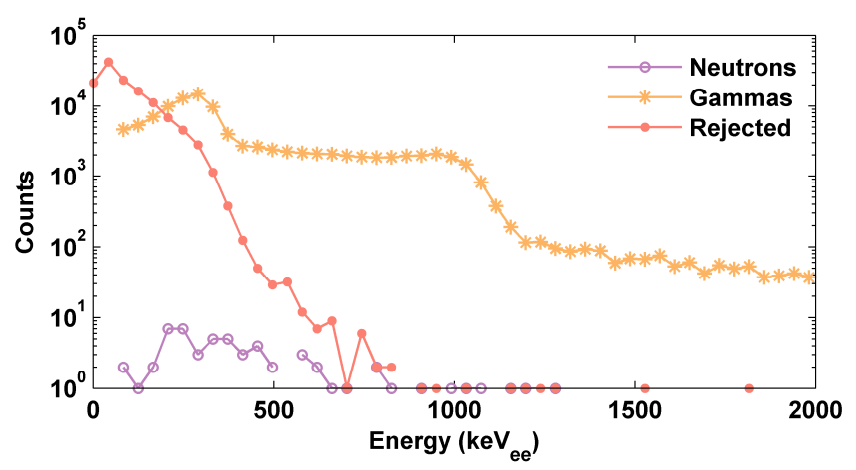

Fig. 9. Separated Na22 spectra from the FB60035 EJ-299-34 detector. The neutron counts measured are misclassified gammas.

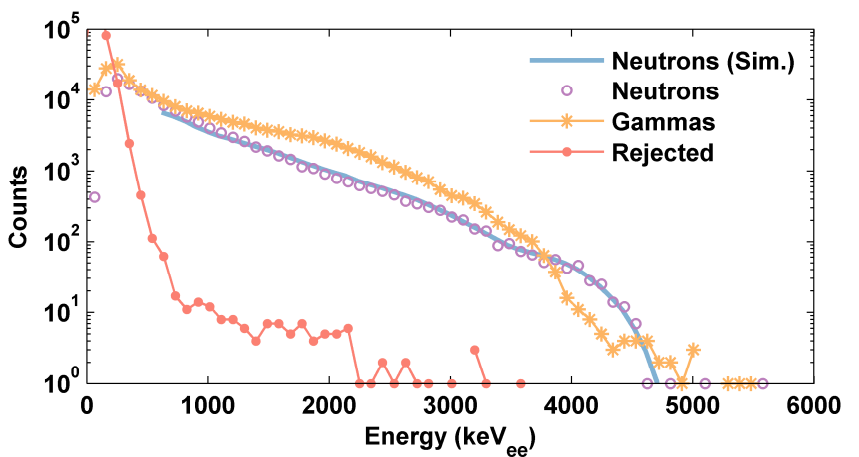

Fig. 10. The simulated neutron spectrum overlaid with the separated AmBe spectra from the FB60035 EJ-299-34 detector.

In-house Monte Carlo neutron transport code, SCIRESP, was used to approximate the plastic scintillator response to the AmBe source. SCIRESP simulates neutron interactions with hydrogen and carbon, the primary mechanisms for fastneutron detection with organic scintillators. The physics model was based on O5R code [43], and the neutron crosssections drawn from ENDF/B-VI [44]. The ISO 8529-1 reference $A m B e$ neutron spectrum was used for the simulated source [40]. The energy deposited by each interaction was converted into an electron-equivalent light yield, depending on the types of charged particles produced, with NE-213 liquid scintillator data used as the surrogate light yield function [45]. The total light yield with each emitted neutron was tallied to give the expected spectrum in $\mathrm{keV}_{\text {ee }}$. The scaled simulated spectrum, overlaid in Fig. 10, showed reasonable agreement with the measured spectrum. The energy scale of the simulation was adjusted by $5 \%$. Discrepancies between the spectra may be due to differences in the light yield functions from EJ-299-34 and NE-213. Furthermore, neutron scattering in the surrounding environment was not included in the simulation. The gamma spectrum includes both gammas emitted by the AmBe source and those due to neutron interactions with the surroundings.

The AmBe data from both detectors was used to assess the acceptance ratio using the defined cuts. The acceptance ratios, shown in Fig. 11, started to degrade once the FOM dropped below 1.27. The acceptance fell to $50 \%$ at $78 \mathrm{keV}_{\text {ee }}$ using stilbene and $185 \mathrm{keV}_{\text {ee }}$ with EJ-299-34.

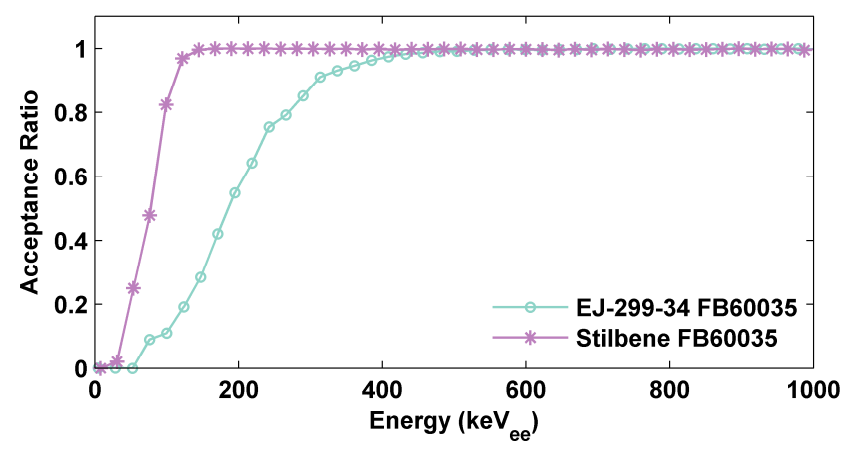

Fig. 11. Acceptance ratio versus energy for the stilbene and EJ-299-34 FB60035 detectors.

\section{PSD Performance at Reduced Digitization Rates}

System performance at lower digitization rates is relevant for developing embedded readout using low-cost hardware. The data sets were decimated to $100 \mathrm{MSps}$ and $50 \mathrm{MSps}$ sampling rates. The original traces were first filtered with a $2^{\text {nd }}$ order Butterworth low-pass filter, the cut-off frequency corresponding to $0.4 \times$ the new sampling frequency. The trace was then down-sampled at the reduced rate. The downsampled data sets were reprocessed using the techniques described above, repeating the pulse alignment, DCC and GA optimization and FOM assessment. The assessed figures of merit at reduced sample rate are shown in Fig. 12 and Fig. 13.

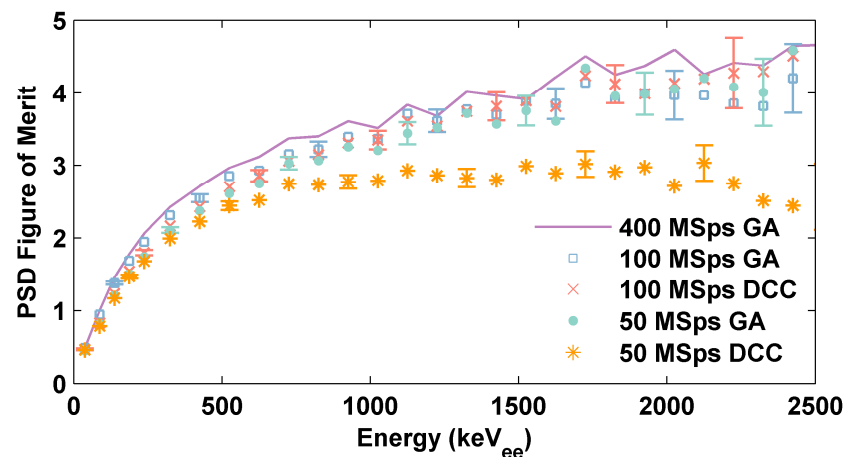

Fig. 12. PSD Figures of Merit measured using the FB60035 stilbene single crystal detector at reduced sample rates. For clarity, only one in every four data points has their $\pm 2 \sigma$ error bars displayed.

At 50 MSps DCC produced significantly reduced figures of merit, while the GA-optimized $\boldsymbol{w}$ maintained performance similar to that measured with the $100 \mathrm{MSps}$ and $400 \mathrm{MSps}$ 
data. The coarse selection of window width available at low sampling rates limits the approximation of the optimum $\boldsymbol{w}$ vector with the flat DCC weights. At $50 \mathrm{MSps}$ the DCC window for both detectors covered two samples. While the GA also gave positive weight to these two samples, the relative values of the weights were considerably different.

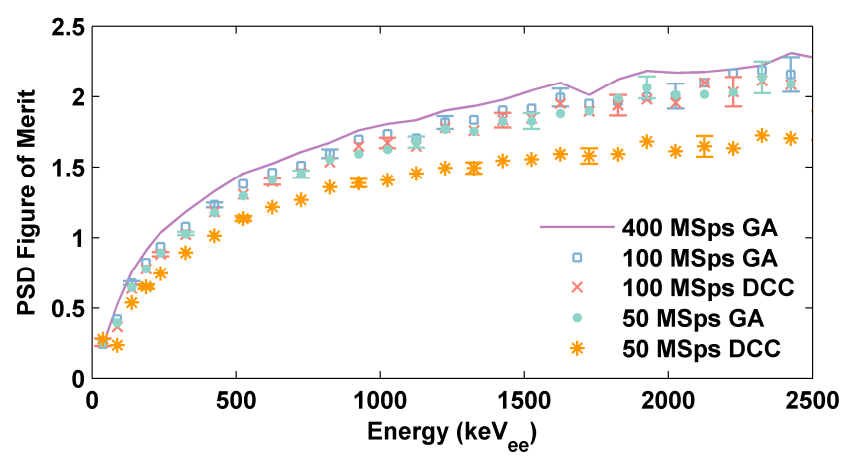

Fig. 13. PSD Figures of Merit measured using the FB60035 EJ-299-34 detector at reduced sampling frequency. For clarity, only one in every four data points has their $\pm 2 \sigma$ error bars displayed.

\section{CONCLUSIONS AND FUTURE WORK}

It was found that organic scintillator detectors with SiPM readout may provide effective discrimination between fast neutron and gamma radiation using digital PSD techniques. The FB60035 SiPM-based detectors provided efficient discrimination down to $127 \mathrm{keV}_{\mathrm{ee}}$ using a stilbene single crystal and $391 \mathrm{keV}_{\text {ee }}$ using an EJ-299-34 developmental PSD plastic scintillator. This was compared with $69 \mathrm{keV}_{\text {ee }}$ and $130 \mathrm{keV}_{\text {ee }}$ measured with an XP2262 PMT. While the SiPM performance was below that measured with a PMT, in many cases the practical and cost benefits of SiPM readout will outweigh the reduced detection efficiency below these energies. A similar argument can be made with the choice of scintillator; whereby stilbene provided superior separation, while the PSD plastic promises to be significantly less expensive, easier to handle, and does not exhibit the significant response anisotropy associated with stilbene [8].

The use of a general purpose digitizer to capture and store detector pulses for later processing in software allowed development and comparison of digital PSD techniques based on weighted integration. A genetic algorithm provided a straightforward approach to optimizing the parameters used for pulse shape measurement. At lower $50 \mathrm{MSps}$ sample rates the GA provided a significant performance advantage over the conventional DCC technique. Meanwhile, DCC delivered near-optimal results when using sample rates at $100 \mathrm{MSps}$ and above. As digital pulse processors capable of DCC are available from a number of manufacturers, DCC provides an attractive off-the-shelf option for performing PSD using SiPM-based detectors.

The respective capabilities of the tested SiPM detectors present an opportunity to apply PSD to a range of new applications where it had not previously been feasible, such as for portable instrumentation and in granular detector-arrays for neutron imaging. To further pursue this notion, we are developing a low-cost and compact digital pulse processor
[46]. The pulse detection, timing, and weighted integration are performed in real-time by a digital signal processor. As the system utilizes a $50 \mathrm{MSps}$ ADC, the GA is used to maximize the PSD performance possible with the hardware.

The EJ-299-34 scintillator, with FB60035 readout and digital pulse processor has been demonstrated as an on-line monitor for pulsed fast-neutron sources used in industry [46]. Another application being explored is the design of a lightweight and portable detector for mixed-field survey and dosimetry. Proton-recoil organic scintillators are relatively efficient at detecting fast neutrons, without the significant mass associated with conventional designs based on the moderation and subsequent capture of neutrons. The plastic detector could be used alone for efficient measurement of fastneutron fields, like the hand-held survey meter developed by Yoshida et al. [47]. Alternatively it could be used in conjunction with a conventional detector, augmenting the detection efficiency for fast-neutrons and reducing the mass of moderator necessary for the desired energy response [48]. The separated fast neutron and gamma spectra may be of additional use in characterizing an unknown radiation field.

\section{ACKNOWLEDGMENT}

The authors would like to thank Charles Hurlbut from Eljen Technology for kindly providing the EJ-299-34 developmental plastic scintillators used in this work.

\section{REFERENCES}

[1] E. Garutti, "Silicon photomultipliers for high energy physics detectors," J. Instrum., vol. 6, no. 10, p. C10003, Oct. 2011. [2] A. Osovizky, D. Ginzburg, A. Manor, R. Seif, M. Ghelman, I. Cohen-Zada, M. Ellenbogen, V. Bronfenmakher, V. Pushkarsky, E. Gonen, T. Mazor, and Y. Cohen, "SENTIRAD—An innovative personal radiation detector based on a scintillation detector and a silicon photomultiplier," Nucl. Instrum. Methods Phys. Res. A, vol. 652, no. 1, pp. 41-44, Oct. 2011.

[3] X. Li, C. Lockhart, T. K. Lewellen, and R. S. Miyaoka, "Study of PET Detector Performance with Varying SiPM Parameters and Readout Schemes.," IEEE Trans. Nucl. Sci., vol. 58, pp. 590-596, Jan. 2011.

[4] V. Golovin and V. Saveliev, "Novel type of avalanche photodetector with Geiger mode operation," Nucl. Instrum. Methods Phys. Res. A, vol. 518, no. 1-2, pp. 560-564, Feb. 2004.

[5] P. Finocchiaro, A. Pappalardo, L. Cosentino, M. Belluso, S. Billotta, G. Bonanno, and S. Di Mauro, "Features of Silicon Photo Multipliers: Precision Measurements of Noise, Cross-Talk, Afterpulsing, Detection Efficiency," IEEE Trans. Nucl. Sci., vol. 56, pp. 1033-1041, Jun. 2009.

[6] R. Bencardino and J. E. Eberhardt, "Development of a Fast-Neutron Detector With Silicon Photomultiplier Readout," IEEE Trans. Nucl. Sci., vol. 56, pp. 1129-1134, Jun. 2009.

[7] R. Preston, J. E. Eberhardt, R. Bencardino, and J. Tickner, "Software-Based Digital Pulse Processing for Silicon Photomultiplier Radiation Detectors," in IEEE NSS/MIC Conf. Rec., 2012, pp. 462-467.

[8] F. D. Brooks, "Development of organic scintillators," Nucl. Instrum. Methods, vol. 162, no. 1-3, pp. 477-505, Jun. 1979.

[9] D. Cester, G. Nebbia, L. Stevanato, F. Pino, L. Sajo-Bohus, and G. Viesti, "A compact neutron-gamma spectrometer," Nucl. Instrum. Methods Phys. Res. A, vol. 719, pp. 81-84, Apr. 2013.

[10] A. G. Wright, "Amplifiers for use with photomultipliers-who needs them?" Nucl. Instrum. Methods Phys. Res. A, vol. 504, no. 1-3, pp. 245-249, May 2003. 
[11] S. Seifert, H. T. van Dam, J. Huizenga, R. Vinke, P. Dendooven, H. Lohner, and D. R. Schaart, "Simulation of Silicon Photomultiplier Signals," IEEE Trans. Nucl. Sci., vol. 56, no. 6, pp. 3726-3733, Dec. 2009.

[12] F. Corsi, A. Dragone, C. Marzocca, A. Del Guerra, P. Delizia, N. Dinu, C. Piemonte, M. Boscardin, and G. F. Dalla Betta, "Modelling a silicon photomultiplier (SiPM) as a signal source for optimum front-end design," Nucl. Instrum. Methods Phys. Res. A, vol. 572, no. 1, pp. 416-418, Mar. 2007.

[13] S. Vinogradov, "Analytical models of probability distribution and excess noise factor of solid state photomultiplier signals with crosstalk," Nucl. Instrum. Methods Phys. Res. A, vol. 695, pp. 247251, Dec. 2012

[14] I. Kreslo, I. Badhrees, S. Delaquis, A. Ereditato, S. Janos, M. Messina, U. Moser, B. Rossi, and M. Zeller, "Pulse-shape discrimination of scintillation from alpha and beta particles with liquid scintillator and Geiger-mode multipixel avalanche diodes," $J$. Instrum., vol. 6, no. 07, p. P07009, Jul. 2011.

[15] B. S. Budden, A. J. Couture, L. C. Stonehill, A. V Klimenko, J. R. Terry, and J. O. Perry, "Analysis of Cs2LiYCl6:Ce3+ (CLYC) waveforms as read out by solid state photomultipliers," in IEEE NSS/MIC Conf. Rec., 2012, pp. 347-350.

[16] J. Glodo, A. Gueorguiev, U. Shirwadkar, R. Hawrami, J. Tower, P. O'Dougherty, and K. S. Shah, "Integrated Neutron Detector for Handheld Systems," IEEE Trans. Nucl. Sci., vol. 60, no. 2, pp. 903907, Apr. 2013.

[17] R. A. Winyard, J. E. Lutkin, and G. W. McBeth, "Pulse shape discrimination in inorganic and organic scintillators. I," Nucl. Instrum. Methods, vol. 95, no. 1, pp. 141-153, Aug. 1971.

[18] N. Zaitseva, B. L. Rupert, I. Pawełczak, A. Glenn, H. P. Martinez, L. Carman, M. Faust, N. Cherepy, and S. Payne, "Plastic scintillators with efficient neutron/gamma pulse shape discrimination," Nucl. Instrum. Methods Phys. Res. A, vol. 668, pp. 88-93, Mar. 2012.

[19] Z.W. Bell, "Tests on a digital neutron-gamma pulse shape discriminator with NE213," Nucl. Instrum. Methods Phys. Res., vol. 188, pp. 105-109, Sep. 1981.

[20] E. Gatti and F. De Martini, "A New Linear Method of Dicrimination Between Elementary Particles In Scintillation Counters," in Proc. Conf. Nucl. Electron., 1962, pp. 15-20.

[21] B. Sabbah and A. Suhami, "An accurate pulse-shape discriminator for a wide range of energies," Nucl. Instrum. Methods, vol. 58, no. 9, pp. 102-110, 1968.

[22] N. V. Kornilov, V. a. Khriatchkov, M. Dunaev, A. B. Kagalenko, N. N. Semenova, V. G. Demenkov, and A. J. M. Plompen, "Neutron spectroscopy with fast waveform digitizer," Nucl. Instrum. Methods Phys. Res. A, vol. 497, no. 2-3, pp. 467-478, Feb. 2003.

[23] S. Marrone, D. Cano-Ott, N. Colonna, C. Domingo, F. Gramegna, E.M. Gonzalez, F. Gunsing, M. Heil, F. Käppeler, P. F. Mastinu, P. M. Milazzo, T. Papaevangelou, P. Pavlopoulos, R. Plag, R. Reifarth, G. Tagliente, J. L. Tain, and K. Wisshak, "Pulse shape analysis of liquid scintillators for neutron studies," Nucl. Instrum. Methods Phys. Res. A, vol. 490, no. 1-2, pp. 299-307, Sep. 2002.

[24] B. D'Mellow, M. D. Aspinall, R. O. Mackin, M. J. Joyce, and A. J. Peyton, "Digital discrimination of neutrons and -rays in liquid scintillators using pulse gradient analysis," Nucl. Instrum. Methods Phys. Res. A, vol. 578, no. 1, pp. 191-197, Jul. 2007.

[25] G. Liu, M. D. Aspinall, X. Ma, and M. J. Joyce, "An investigation of the digital discrimination of neutrons and $\gamma$ rays with organic scintillation detectors using an artificial neural network," Nucl. Instrum. Methods Phys. Res. A, vol. 607, no. 3, pp. 620-628, Aug. 2009.

[26] S. Yousefi, L. Lucchese, and M. D. Aspinall, "Digital discrimination of neutrons and gamma-rays in liquid scintillators using wavelets," Nucl. Instrum. Methods Phys. Res. A, vol. 598, no. 2, pp. 551-555, Jan. 2009.

[27] D. I. Shippen, M. J. Joyce, and M. D. Aspinall, "A Wavelet Packet Transform Inspired Method of Neutron-Gamma Discrimination," IEEE Trans. Nucl. Sci., vol. 57, pp. 2617-2624, Oct. 2010.

[28] G. Liu, M. J. Joyce, X. Ma, and M. D. Aspinall, “A Digital Method for the Discrimination of Neutrons and Gamma Rays With Organic Scintillation Detectors Using Frequency Gradient Analysis," IEEE Trans. Nucl. Sci., vol. 57, pp. 1682-1691, Jun. 2010.
[29] V. Jordanov and G. Knoll, "Digital pulse-shape analyzer based on fast sampling of an integrated charge pulse," IEEE Trans. Nucl. Sci., vol. 42, pp. 683-687, Aug. 1995.

[30] P.-A. Söderström, J. Nyberg, and R. Wolters, "Digital pulse-shape discrimination of fast neutrons and y rays," Nucl. Instrum. Methods Phys. Res. A, vol. 594, no. 1, pp. 79-89, Aug. 2008.

[31] E. Gatti and F. De Martini, "Optimum linear filter for pulse shape recognition in the presence of thermal noise," Energ. Nucl., vol. 9, no. 3, Mar. 1962.

[32] J. H. Holland, Adaption in natural and artificial systems, Ann Arbor: MIT Press, 1992.

[33] K. De Jong, "Learning with genetic algorithms: An overview," Mach. Learn., vol. 3, no. 2-3, pp. 121-138, 1988.

[34] K. De Jong, "Analysis of the behavior of a class of genetic adaptive systems," Ph.D. dissertation, Dept. Comput. Sci., Univ. Michigan, Ann Arbor, MI, 1975.

[35] L. J. Eshelman and D. J. Schaffer, "Real-coded Genetic Algorithms and Interval-Schemata," in Foundations of Genetic Algorithms 2. San Mateo, CA, 1993, pp. 187-202

[36] J. E. Baker, "Adaptive Selection Methods for Genetic Algorithms," in Proc. of the 1st Int. Conf. on Genetic Algorithms, Pittsburgh, PA, 1985, pp. 101-111.

[37] N. Radcliffe, "Equivalence class analysis of genetic algorithms," Complex Syst., vol. 5, pp. 183-205, 1991.

[38] A. Favalli, M. L. Iliev, K. Chung, C. Hurlbut, H. P. Martinez, M. T. Swinhoe, N. P. Zaitseva, and K. D. Ianakiev, "Pulse Shape Discrimination Properties of Neutron-Sensitive Organic Scintillators," IEEE Trans. Nucl. Sci., vol. 60, pp. 1053-1056, Apr. 2013.

[39] G. Ranucci, R. Dossi, P. Inzani, G. Korga, P. Lombardi, E. Meroni, and M. E. Monzani, "A sampling board optimized for pulse shape discrimination in liquid scintillator applications," IEEE Trans. Nucl. Sci., vol. 51, pp. 1784-1790, Aug. 2004.

[40] Reference Neutron Radiations - Part 1, ISO 8529-1, 2000.

[41] H. R. Vega-Carrillo, E. Manzanares-Acuña, A. M. Becerra-Ferreiro, and A. Carrillo-Nuñez, "Neutron and gamma-ray spectra of 239PuBe and 241 AmBe," Appl. Radiat. Isot., vol. 57, no. 2, pp. 167-170, Aug. 2002.

[42] D. Smith, R. Polk, and T. Miller, "Measurement of the response of several organic scintillators to electrons, protons and deuterons," Nucl. Instrum. Methods, vol. 64, no. 2, pp. 157-166, 1968.

[43] R. R. Coveyou, J. G. Sullivan, H. P. Carter, D. C. Irving, R. M. Freestone Jr, and F. B. K. Kam, "O5R, a General-Purpose Monte Carlo Neutron Transport Code," ORNL, Oak Ridge, TN, Rep. ORNL-3622, Feb. 1965.

[44] P. F. Rose, "ENDF/B-VI summary documentation", NNDC, BNL, Upton, NY, Rep. BNL-NCS-17541, 1991.

[45] V. Verbinski, W. Burrus, and T. Love, "Calibration of an organic scintillator for neutron spectrometry," Nucl. Instrum. Methods, vol. 65, 1968 .

[46] R. M. Preston, J. E. Eberhardt, and J. R. Tickner, "Neutron generator burst timing measured using a pulse shape discrimination plastic scintillator with silicon photomultiplier readout," J. Instrum. vol. 8, no. 12, p. P12005, Dec. 2013.

[47] T. Yoshida, N. Tsujimura, and T. Yamano, "Development of a hand-held fast neutron survey meter.," Radiat. Prot. Dosimetry, vol. 146, no. 1-3, pp. 72-5, Jul. 2011.

[48] R. Olsher, D. Seagraves, S. Eisele, C. Bjork, W. Martinez, L. Romero, M. Mallet, M. Duran, and C. Hurlbut, "PRESCILA: a new, lightweight neutron rem meter," Health Phys., vol. 86, no. 6, pp. 603-612, Jun. 2004. 